\title{
Implante percutáneo de válvula aórtica con apoyo de circulación extracorpórea en paciente con insuficiencia aórtica severa
}

\author{
Gonzalo Martínez, Martín Valdebenito, Samuel Córdova, Ramón Corbalán, Guillermo Lema, Rodrigo \\ Bahamondes, Alejandro Martínez, Ricardo Zalaquett. \\ División de Enfermedades Cardiovasculares, Pontificia Universidad Católica de Chile \\ Departamento de Anestesiología, Pontificia Universidad Católica de Chile
}

\section{Trans catheter aortic valve implantation with extra corporeal circulation support in a patient with severe aortic insufficiency}

An 84-year man with prior coronary artery bypass surgery and endovascular repair of an abdominal aortic aneurysm developed congestive heart failure. He had calcific aortic valve disease with severe regurgitation. A \#29 Edwards-Sapien aortic valve via trans-apical approach was implanted with the patient connected to extra corporeal circulation. The patient recovered successfully and remained in functional class II 8 months after hospital discharge.

Key words: Aortic insufficiency; trans aortic valve implantation; extracorporeal circulation.

\section{Correspondencia:}

Dr. Gonzalo Martínez R.

gjmartin@uc.cl

Departamento de Enfermedades Cardiovasculares,

Pontificia Universidad Católica de Chile, Marcoleta 367, $2^{\circ}$ piso. 


\section{Cuadro clínico}

Paciente de 84 años, con antecedentes de cirugía de revascularización miocárdica 14 años atrás, aneurisma de aorta abdominal tratado con endoprótesis hace 1 año, insuficiencia renal crónica en etapa IV, enfermedad vascular periférica y ex tabáquico. Consultó por cuadro progresivo de insuficiencia cardíaca, llegando a capacidad funcional IV de NYHA en las semanas previas. Se hospitalizó y su ecocardiograma mostró un ventrículo izquierdo hipertrófico, con disfunción sistólica moderada y fracción de eyección de $38 \%$, una secuela de infarto antiguo en paredes inferior e inferolateral, junto a un ventrículo derecho dilatado, disfuncionante, con hipertensión pulmonar severa (presión sistólica de arteria pulmonar $80 \mathrm{mmHg}$ ). El estudio de la válvula aórtica mostró calcificación leve y asimétrica de los velos aórticos, con velo no coronariano fijo, lo que producía una insuficiencia aórtica severa y estenosis moderada (Vmax $3,2 \mathrm{~m} / \mathrm{s}$, gradiente media $42 \mathrm{mmHg}$, área valvular 1,2 cm2). La válvula mitral presentaba una insuficiencia moderada secundaria a "tenting" del aparato subvalvular. Su creatinina plasmática era $1,6 \mathrm{mg} / \mathrm{dL}$, el hematocrito $31 \%$ y NT-pro-BNP 12.282 $\mathrm{ng} / \mathrm{ml}$. La coronariografía mostró puentes permeables (Arteria mamaria izquierda a la ADA (LIMA-DA), puente venoso en Y a la arteria circumfleja (PAC-Cx en Y) y puente venoso a coronaria derecha (PAC CD), con enfermedad de sus tres vasos nativos. Una aortografía confirmó el hallazgo de insuficiencia aórtica severa (Figura 1).

El paciente fue manejado inicialmente con terapia farmacológica. Sin embargo, pocas semanas luego de su alta nuevamente presentó síntomas progresivos de insuficiencia cardíaca, recayendo en capacidad funcional IV, con marcado edema periférico. En ese contexto se evaluó para eventual implante percutáneo de prótesis aórtica (TAVR). Se realizó un AngioTAC (Figura 2) que mostró el aneurisma de aorta abdominal, con endoprótesis permeable, sin endoleak, junto a marcada tortuosidad de aorta abdominal y ateromatosis mixta severa de arterias ilíacas externas y femorales.

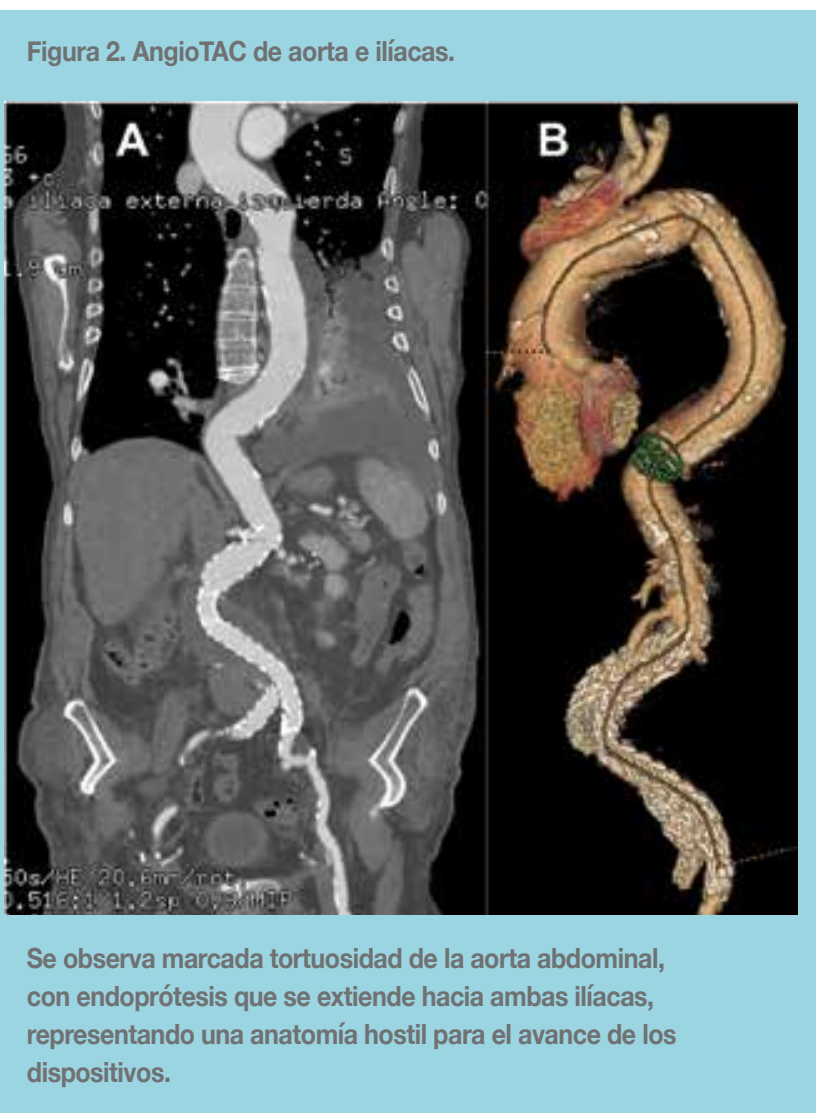

Figura 1. Insuficiencia aórtica severa.

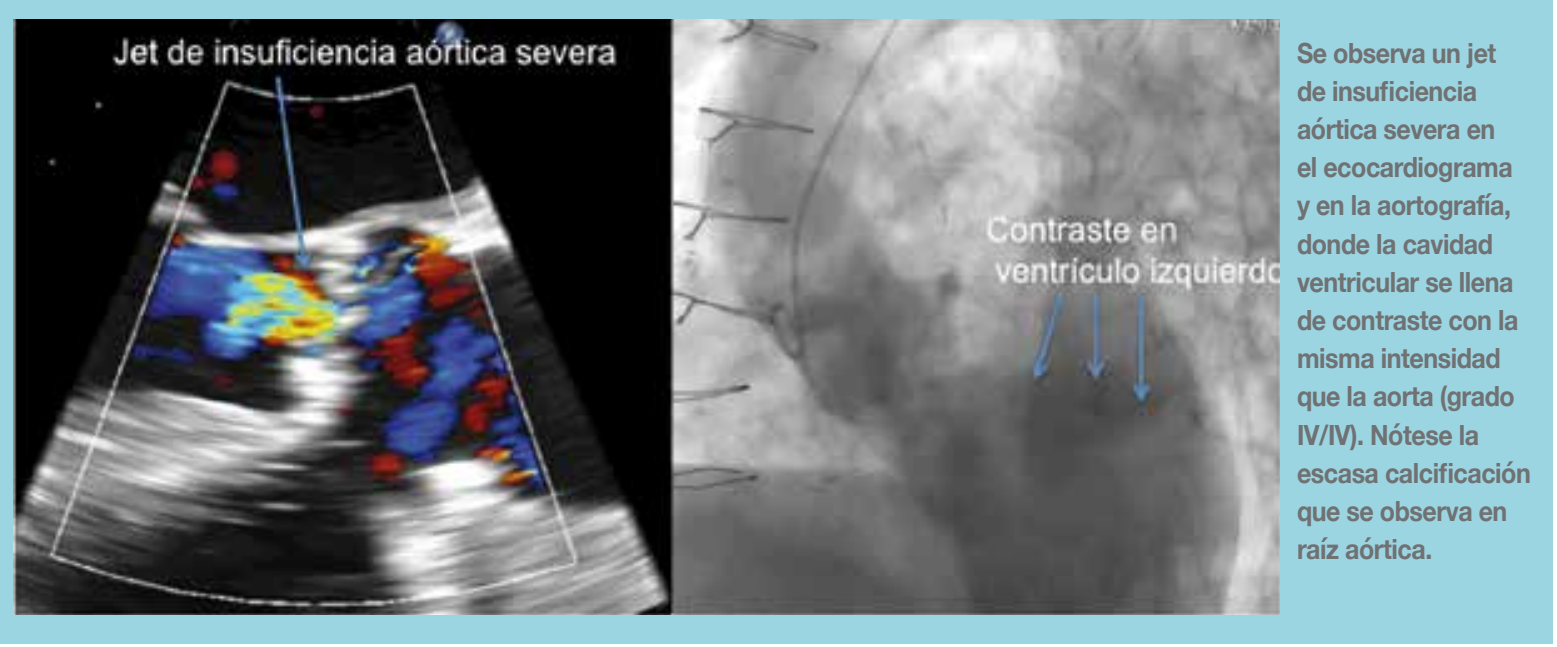


Las mediciones del anillo valvular aórtico fueron: Diámetro 27x24mm, Perímetro 83mm, Área $540 \mathrm{~mm} 2$ y Diámetro derivado de perímetro $26,4 \mathrm{~mm}$.

Adicionalmente, se calculó un EuroSCORE II de 42,33\% y STS Score de $17,17 \%$. El paciente fue evaluado en forma multidisciplinaria por nuestro "Heart Team" y se concluyó que su riesgo era prohibitivo para realizar cirugía convencional, dado que sumado al riesgo estimado por los scores tradicionales presentaba un alto índice de fragilidad. El equipo identificó además que este paciente presentaba una serie de condiciones que podían aumentar el riesgo del implante percutáneo y que requerían especial atención:

1. Presencia de insuficiencia como enfermedad predominante de su válvula aórtica, lo cual aumenta el riesgo de mal posicionamiento.

2. Severa tortuosidad y enfermedad del complejo aorto-ilíaco, con endoprótesis en aorta abdominal, descartando el acceso transfemoral.

3. Hipertensión pulmonar severa, con disfunción de ventrículo derecho, lo cual puede determinar shock irreversible luego del implante de la prótesis.

4. Disfunción ventricular izquierda, con sobrecarga de volumen. Dado que su patología predominante era la insuficiencia, se estimó posible que la recuperación inmediata post implante que se observa habitualmente en las estenosis, no ocurriera en este caso, complicando su evolución precoz.

Otros accesos posibles, como trans-aórtico o subclavio izquierdo, fueron descartados por la presencia de puentes venosos y puente mamario, respectivamente, que potencialmente podrían dañarse con la inserción del introductor. El acceso subclavio derecho fue desechado por la presencia de un ángulo desfavorable para el implante de la prótesis en la llegada del tronco braquiocefálico. Considerando todos estos aspectos, se decidió realizar el implante de una prótesis Edwards-Sapien \#29 por vía trans-apical, con el apoyo de oxigenador de membrana extracorpóreo (ECMO) arterio-venoso durante el implante.

\section{Implante trans-apical de prótesis aórtica.}

El procedimiento fue realizado en sala híbrida con la participación de un equipo multidisciplinario compuesto por cardiólogos intervencionales, cirujano cardíaco, anestesista cardiovascular, ecocardiografista y perfusionista, junto al equipo de enfermería y técnicos del laboratorio. El paciente fue posicionado en decúbito supino. Luego de inducida la anestesia general endotraqueal, se colocó una línea arterial radial derecha, una sonda marcapaso transitoria introducida por vía yugular izquierda y un catéter de Swan-Ganz introducido a través de la vena yugular derecha. Con técnica quirúrgica se accedieron la arteria y vena femoral izquierda para introducir las cánulas de circulación extracorpórea (14F arterial, 20F venoso) (Figura 3). Desde este momento, el procedimiento fue asistido

Figura 3.

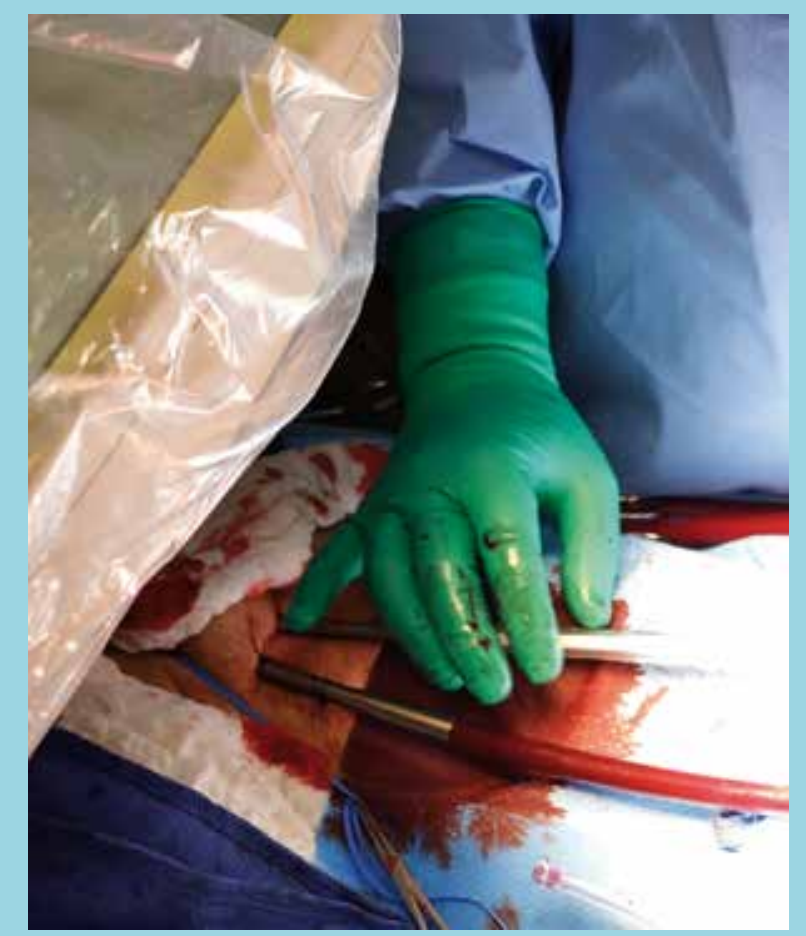

Canulación femoral izquierda arterial y venosa para ECMO.

con soporte parcial por ECMO. Por medio de un acceso arterial femoral derecho $6 \mathrm{~F}$ se posicionó un catéter pigtail en la raíz aórtica. Luego, se efectuó una toracotomía anterior izquierda de aproximadamente 10 centímetros a nivel del $5^{\circ}$ espacio intercostal el que fue definido previamente de acuerdo al angioTAC de tórax. Abierto el saco pericárdico y liberada la zona anteroapical del corazón, se procedió a colocar 2 puntos de Prolene 2-0 en U perpendiculares entre sí a aproximadamente 2 a 3 centímetros del ápex del corazón y del surco interventricular, para efectuar la punción del ventrículo izquierdo. El sitio de punción fue ratificado bajo visión ecocardiográfica, deprimiendo digitalmente la pared del ventrículo izquierdo. Se avanzó una guía 0,035 " de forma anterógrada a través de la válvula aórtica, posicionándola hasta la aorta 
descendente. Esta guía fue intercambiada por una guía Amplatz ${ }^{\circledR}$ Extra Stiff, sobre la cual se avanzó el introductor ASCENDRA+ + , posicionándolo $4 \mathrm{~cm}$ en la cavidad ventricular izquierda. A través de éste se avanzó una prótesis aórtica Edwards-Sapien de $29 \mathrm{~mm}$, que se posicionó en el anillo aórtico con ayuda fluoroscópica y ecocardiográfica. Dada la escasa calcificación identificable en fluoroscopía, el apoyo del eco transesofágico resultó fundamental para el posicionamiento de la prótesis. En ese momento se pasó a soporte completo con ECMO y realizó estimulación rápida con marcapasos, la cual pudo ser mantenida por alrededor de 45 segundos gracias al soporte hemodinámico, desplegando la prótesis una vez seguros de su posición correcta (Figura 4).

Figura 4. Prótesis Edwards-Sapien \#29 en posición aórtica.

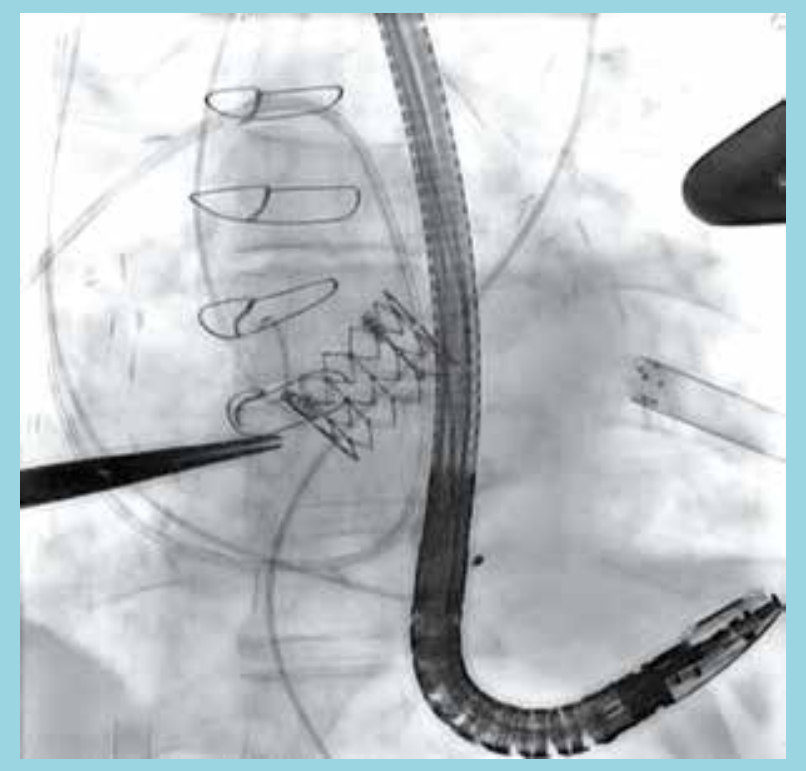

La evaluación post-implante angiográfica y ecocardiográfica no evidenció regurgitación luego del implante.

Tanto la evaluación ecocardiográfica como la aortografía mostraron una excelente posición de la prótesis, sin evidencias de leak paravalvular relevante y con gradientes adecuadas. Con este resultado, se procedió a cerrar el acceso apical.

El soporte con ECMO se mantuvo por algunos minutos luego del implante, reduciéndose progresivamente y finalmente suspendiéndose antes de salir de pabellón, lo cual permitió su decanulación inmediata.

El paciente fue derivado a la unidad de recuperación car- diovascular, siendo extubado el mismo día. Su mejoría fue progresiva, lo que permitió el alta 10 días después del procedimiento.

Luego de 8 meses desde la intervención, el paciente se ha mantenido en buenas condiciones generales, en CF II, y no ha requerido hospitalizaciones ni nuevas intervenciones. Su función renal presentó una mejoría significativa, estabilizándose en valores de clearance de creatinina de $40 \mathrm{ml} / \mathrm{min}$.

\section{Discusión}

TAVR se ha transformado en la técnica de elección para pacientes con estenosis aórtica severa calcificada en quienes se estima que su riesgo quirúrgico es muy alto o prohibitivo para cirugía convencional, especialmente cuando el TAVR se puede realizar por vía transfemoral ${ }^{1}$. En el caso que presentamos, sin embargo, la patología predominante era insuficiencia aórtica, para lo cual esta tecnología no fue específicamente diseñada. Adicionalmente, la condición del paciente era crítica, en falla cardíaca, con compromiso severo de sus cavidades derechas e hipertensión pulmonar.

Publicaciones previas han sugerido algunas condiciones donde el uso profiláctico de ECMO puede ser de utilidad $^{2}$ :

- Insuficiencia cardíaca que requiere hospitalización y estabilización pre-TAVR.

- Disfunción moderada a severa de ventrículo derecho y/o severa de ventrículo izquierdo.

- Parámetros hemodinámicos alterados, que incluyen PVC $>20 \mathrm{mmHg}$, PAPm. $>40 \mathrm{mmHg}$, índice cardÍaco $<2$ 1/min/m2 sin mejoría con inótropos.

- Inestabilidad hemodinámica durante una balón plastia aórtica realizada previamente.

De éstas, nuestro paciente presentaba al menos tres, sumado a que su patología predominante correspondía a insuficiencia aórtica, con escasa calcificación del anillo, lo cual podía hacer el implante de la prótesis difícil, con posibilidad de mal-posicionamiento ${ }^{3}$.

Seco y cols2 publicaron la experiencia de 11 pacientes en los cuales se realizó TAVR con apoyo de ECMO: 8 fueron conectados de forma profiláctica y 3 como rescate ante complicaciones del procedimiento. Es importante que aquellos pacientes conectados de forma profiláctica presentaron $0 \%$ de mortalidad al mes, a pesar de un alto riesgo pre-intervención, documentado por un Euroscore I $(\log )$ de $49 \pm 24$. Dos pacientes presentaron fibrila- 
ción ventricular, mal-posicionamiento de prótesis en 1, sangrado con riesgo vital en 1 y complicación vascular mayor en 1 . Drews y cols ${ }^{4}$ reportaron el uso de circulación extracorpórea femoro-femoral durante TAVR en 43 pacientes. Las principales razones para usarlo fueron 1) hipertensión pulmonar combinada con crecimiento o disfunción de cavidades derechas o disfunción severa de ventrículo izquierdo en 20 pacientes, para prevenir la inestabilidad hemodinámica durante el implante de la válvula o en caso de arritmias ventriculares; 2) para descargar las presiones ventriculares y promover la recuperación cardiaca en pacientes con shock cardiogénico, en 23 casos. Es interesante que en aquellos pacientes con la primera indicación (similar a la usada en nuestro caso), la sobrevida a 30 días y a 1 año fue de $100 \%$ y $86 \%$, respectivamente, en cambio en aquellos con shock car- diogénico el pronóstico fue mucho peor.

El presente caso demuestra como el uso de soporte hemodinámico veno-arterial puede ser de utilidad para pacientes de alto riesgo, en particular en aquellos con hipertensión pulmonar y disfunción de ventrículo derecho o en quienes no se espera una recuperación inmediata luego del implante. En nuestra institución, TAVR es realizada por un equipo multidisciplinario, que incluye la colaboración estrecha entre cardiólogos intervencionales, cirujanos cardiacos, ecocardiografistas y anestesistas cardiovasculares, tanto en la planificación como en la ejecución de los procedimientos. Esta organización nos parece importante para poder concretar de forma segura y eficiente procedimientos complejos que puedan beneficiar a nuestros pacientes.

Los autores declaran no tener conflictos de interés.

\section{Referencias}

1- GÉNÉREUX P, HEAD SJ, WOOD DA, KODALI SK, WILLIAMS MR, PARADIS JM, et al. Transcatheter aortic valve implantation 10-year anniversary: review of current evidence and clinical implications. Eur Heart J. 2012; 33: 2388-98.

2- SECO M, FORREST P, JACKSON SA, MARTÍNEZ G, ANDVIK S, BANNON PG, et al. Extracorporeal membrane oxygenation for very high-risk transcatheter aortic valve implantation. Heart Lung Circ. 2014; 23: 957-62.
3- ROY DA, SCHAEFER U, GUETTA V, HILDICK-SMITH D, MÖLLMANN H, DUMONTEIL N, et al. Transcatheter aortic valve implantation for pure severe native aortic valve regurgitation. J Am Coll Cardiol. 2013; 61: 1577-84.

4- DREWS T, PASIC M, BUZ S, DREYSSE S, KLEIN C, KUKUCKA M, et al. Elective use of femoro-femoral cardiopulmonary bypass during transcatheter aortic valve implantation. Eur J Cardiothorac Surg. 2015; 47: 24-30. 